\title{
Referees 2008
}

The quality of the Journal of Orthopaedics and Traumatology depends on the qualified and regular collaboration of renowned scientists who devoted their time to

Paolo Arrigoni (Milan, Italy)

Pietro Bartolozzi (Verona, Italy)

Celeste Bertone (Brescia, Italy)

Amin Sadegh Bigham (Shahrekord, Iran)

Sergio Brambilla (Milan, Italy)

Umberto de Bellis (Milan, Italy)

Shabir A. Dhar (Srinagar, India)

Cesare Faldini (Bologna, Milan)

Olimpio Galasso (Catanzaro, Italy)

Vincenzo Guzzanti (Rome, Italy)

Yoichi Koike (Miyagi, Japan)

Arun Kumar (Cheshire, UK)

Harish V. Kurup (Croydon, UK)

Giulio Maccauro (Rome, Italy)

Bruno Magnan (Verona, Italy) constructively review the submitted articles. We are indebted to the following experts who reviewed papers which completed the peer-reviewing process within 2008.

Masahiko Nozawa (Tokyo, Japan)

Matthew E. Oetgen (New Haven, CT, USA)

Elisha Ofiram (Rehovot, Israel)

Roberto Padua (Rome, Italy)

Luca Pierannunzii (Milan, Italy)

Filippo Randelli (Milan, Italy)

Enrico Rebuzzi (Oderzo, Italy)

Fabrizio Rivera (Turin, Italy)

Celeste Scotti (Milan, Italy)

Kei Shiramizu (Sydney, Australia)

Roop Singh (Rohtak, India)

Matthias Therbo (Copenhagen, Denmark)

Paolo Tranquilli Leali (Sassari, Italy)

Carmine Zoccali (Rome, Italy) 